Psychological Medicine, 2001, 31, 761-767. Printed in the United Kingdom

(C) 2001 Cambridge University Press

EDITORIAL

\title{
Acute sickness behaviour: an immune system-to-brain communication? ${ }^{1}$
}

Over the past 20 years, psychoneuroimmunological research has produced a large body of evidence that challenges the historically dominant view that the immune system operates in an autonomous manner independent of other physiological systems. Today, there is little doubt that the brain and the immune system are intimately linked and capable of reciprocal communication (Ader et al. 1991). Despite the acknowledged bi-directional nature of the brain-immune system connection, the predominant focus of study has been on the effects of psychological and behavioural events (e.g. stress) on immune responses and disease processes, and the mechanisms underlying such effects (see Kusnekov \& Rabin, 1994; Maier et al. 1994; Rozlog et al. 1999). However, considerable interest in the possibilities of immune-system-to-brain communication was initiated by a seminal paper considering the biological basis of behaviour in sick animals (Hart, 1988). Subsequently, the immunological determinants of the behavioural, cognitive and emotional changes associated with acute illness, as well as with more chronic psychopathological states (e.g. depression) have become the subject of rapidly expanding areas of research (e.g. Kent et al. 1992; Lloyd et al. 1992; Hickie \& Lloyd, 1995; Maes et al. 1995 a Rothwell \& Hopkins, 1995; Dantzer et al. 1996; Maier \& Watkins, 1998; Vollmer-Conna et al. 1998; Maes, 1999).

The main objective of this editorial is to provide a succinct overview of current knowledge of the normal behavioural correlates of acute infective illness, their adaptive function and underlying mechanisms. Elucidation of the processes involved in the appearance, maintenance and inhibition of 'normal' sickness behaviour is important if extrapolations from this phenomenon to more chronic psychopathological conditions are to provide more than a new label for poorly understood non-specific symptom clusters.

\section{A ROLE FOR SICKNESS BEHAVIOUR IN THE HOST DEFENCE AGAINST INFECTION AND INFLAMMATION}

Acute infective illnesses, both in animals and humans, are typically accompanied by a cluster of non-specific symptoms such as fever, an increased need to sleep, hyperalgesia, anorexia, loss of interest in usual activities, decreased social interaction and body care, depression and impaired concentration (Hart, 1988; Dantzer et al. 1996). Perhaps because they are prevalent and nonspecific concomitants of infective illnesses, these phenomena are commonly dismissed or relatively ignored by physicians. Yet, it has recently been argued that sickness behaviours constitute a highly organized and evolved strategy to combat infection and injury. Specifically, it has been suggested that the behavioural changes function to conserve energy and, thus, facilitate the role of fever in stimulating immune function and inhibiting the proliferation of thermo-sensitive pathogens (Hart, 1988).

The adaptive nature of the fever response is well documented and apparent from numerous demonstrations that inhibition of fever (e.g. by placing infected animals in a cold environment, or treating them with antipyretic drugs) is detrimental to survival (Kluger, 1979). Interference with sickness behaviours, by force-feeding or sleep-depriving animals with acute infections, similarly produces a marked reduction in survival rates (Hart, 1988). Moreover, there is evidence to suggest

1 Addressforcorrespondence:DrUtéVollmer-Conna,DepartmentofHumanBehaviour,SchoolofPsychiatry,UniversityofNewSouth Wales, UNSW Sydney 2052, Australia. 
that sickness behaviour is not merely an automatic, inflexible reaction to illness, but rather the expression of a central motivational state. That is, animals will actively learn responses to be able to engage in these behaviours when they are infected and will choose conditions under which they can occur (Dantzer et al. 1996; Maier \& Watkins, 1999).

\section{ACUTE SICKNESS BEHAVIOUR IS IMMUNOLOGICALLY MEDIATED}

The production of the pro-inflammatory cytokines such as tumour necrosis factor (TNF), interleukin-1 (IL-1) and IL-6 by activated immune cells (monocyte/macrophages, lymphocytes) is an integral part of the host response to infection. These cytokines act as messenger molecules and play a pivotal role in the orchestration of the acute phase response (Dinarello, 1997; Papanicolaou, 1998). In recent years, it has also become clear that the necessary synchrony between metabolic, physiological and behavioural aspects of the individual's response to infection depends on the activities of these same cytokines. It has long been known that fever is not caused directly by invading pathogens but rather by the action of soluble immunological products. These endogenous pyrogens were subsequently identified as being pro-inflammatory cytokines, with IL-1 the most potent pyrogenic agent (Dinarello et al. 1977). The first indication of a role for cytokines in the induction of acute sickness behaviour came from clinical trials with purified or recombinant cytokines. Administration of cytokines in the treatment of cancer and chronic viral infections such as hepatitis B and C produced a syndrome of adverse effects, including fever, fatigue, malaise, headaches, anorexia, depression and, at high doses, delirium (Renault \& Hoofnagel, 1989; Dinarello, 1997). However, as these clinical observations were made predominantly on patients with significant medical illnesses, generalization from these findings to argue for a role for cytokines in normal sickness behaviour is problematical.

Animal experiments have confirmed that most aspects of sickness behaviour can be induced in a dose-dependent fashion by systemic or intracerebral injections of pro-inflammatory cytokines, particularly IL-1 $\beta$ (Dantzer et al. 1996). Systemic injections with lipopolysaccharide (LPS), a strong inducer of the synthesis and release of most pro-inflammatory cytokines, also produces the full spectrum of sickness behaviour. Conversely, administration of specific antagonists (e.g. IL-1 receptor antagonist (IL-1Ra)) inhibits many of the central effects of this cytokine (Rothwell \& Hopkins, 1995). Given the pleiotropism and redundancy of the cytokine network, it has been difficult to determine the precise contribution of individual cytokines to sickness behaviour. However, there is evidence to suggests that TNF- $\alpha$ and IL-1- $\beta$, which are synthesized very early in the immune response, are more potent than those that are induced later (e.g. IL-6) and act in synergy to produce sickness behaviour (Dantzer et al. 1996). IL-6 appears to require the presence of other pro-inflammatory cytokines (e.g. IL-1) to produce behavioural symptoms of sickness (Bluthe et al. 1998). The role of IL-6 in sickness behaviour is likely to be complex, as recent evidence suggests that IL-6 has both pro- and anti-inflammatory functions, the latter including the induction of natural antagonists to TNF and IL-1 and stimulation of the hypothalamic-pituitary-adrenal (HPA) axis (Papanicolaou, 1998).

\section{LINKS BETWEEN ACUTE SICKNESS BEHAVIOUR AND THE STRESS RESPONSE}

Experimental administration of pro-inflammatory cytokines or LPS to animals has revealed that, in addition to acute sickness behaviour, a classic stress response is produced. This is characterized by activation of the sympathetic nervous system and release of plasma catecholamines as well as activation of the HPA system leading to the release of adrenocorticotrophic hormone (ACTH) and glucocorticoids (Dunn, 1995; Maier \& Watkins, 1999). In humans, IL-6 appears to be a particularly potent stimulator of the HPA axis. For example, daily administration of recombinant IL-6 over a week was found to produce a remarkable activation and enlargement of the adrenal glands, similar to that seen in patients with Cushing's disease (Mastorakos et al. 1993). Because glucocorticoids, in turn, exert negative feedback on the secretion of IL-6, it has been argued that this cytokine displays the traditional characteristics of a hormone (Papanicolaou, 1998). 
It is well established that exposure to stressors may result in the suppression of cell-mediated immune responses (Kusnekov \& Rabin, 1994). However, new evidence suggests that the sequelae of exposure to acute stressors are considerably more complex than previously thought and include aspects of immune activation not unlike the host response to acute infections (Maier \& Watkins, 1998; Maes, 1999; Tringali et al. 2000, for reviews). Briefly, in both humans and animals, diverse physical and psychosocial stressors were found to produce leucocytosis, a shift in liver metabolism toward production of acute phase proteins, secretion of pro-inflammatory cytokines and fever. Moreover, behavioural changes including anorexia, decreases in activation, exploration, social interaction and aggression, depressed affect and cognitive impairment, bear a strong resemblance to cytokine-induced sickness behaviour and appear more adaptive in the context of sickness then as a component of the fright-fight response.

The similarities between aspects of the stress response and acute sickness behaviour point to a common mediator. Indeed, a direct role for pro-inflammatory cytokines in the stress response was established by experiments blocking specific cytokine receptors in the brain during exposure to a stressor. For example, injections of the IL-1 receptor antagonist (IL-1Ra) abolished the learned helplessness response and exaggerated fear typically induced by inescapable shock (Maier \& Watkins, 1995), as well as blunting the pituitary-adrenal response and inhibiting the release of hypothalamic monoamines normally associated with immobilization stress (Shintani et al. 1995). There is also evidence that the stress-induced secretion of cytokines in hypothalamic structures and the periphery are mediated by catecholaminergic mechanisms such as the activation of $\beta$-adrenergic receptors (Takaki et al. 1994; Papanicolaou, 1998; Tringali et al. 2000).

There remains little doubt that the systems mediating the organism's defence against infection and injury on the one hand, and against psychosocial and environmental stressors on the other, are closely connected. Indeed, it has been proposed that environmental (i.e. 'external') stressors essentially activate the same neuro-endorine-immune circuitry as immunogenic agents (i.e. 'internal' stressors), although they may enter the circuit at different sites (Maier \& Watkins, 1998; Maes, 1999). The end product of this activation (in terms of neuroendocrine, behavioural, cognitive, emotional, or immunological consequences - which are clearly different for different trigger stimuli) presumably depends on the specific characteristics of the trigger (Maier \&Watkins, 1998; Tringali et al. 2000).

From an evolutionary perspective, the similarities in the response to stress and acute illness may be understood by studying the systems subserving adaptation and defence in primitive organisms. Even in the most primitive organisms such as molluscs and sponges, which are incapable of elaborate defence against distal threats, some form of host response against infection and injury can be identified (Beck et al. 1994). Moreover, there is evidence that as early in evolution as the molluscs, defence against infection and injury involved pro-inflammatory cytokines in bi-directional communication between immunological and neural structures, as well as the release peptides traditionally viewed as stress hormones (Clatworthy, 1996; Maier \& Watkins, 1998, for reviews). When the fright-flight response evolved later in more complex organisms, existing mechanisms such as cytokine-based communication networks appear to have been incorporated in this new adaptation (Maier \& Watkins, 1998). This account, although speculative, offers an explanation for the mobilization of inflammatory processes during acute stress, and provides a basis for the manifestation of seemingly maladaptive behavioural changes (i.e. sickness behaviour) after exposure to a significant stressor.

\section{POSSIBLE MECHANISMS UNDERLYING THE PRODUCTION OF ACUTE SICKNESS BEHAVIOUR}

In view of their documented involvement in diverse centrally-mediated phenomena, there is little doubt that cytokines are capable of providing signals to the brain to alter neural activity. The mechanism through which these peripherally produced molecules might act on the brain, however, has been the subject of much debate. Cytokines are not thought to cross the blood-brain barrier, 
owing to their large molecular weight and hydrophilic properties. A number of specialized mechanisms have been identified, however, that would allow blood-borne cytokines to signal the brain. These include an active transport system across the barrier (Banks et al. 1991), and cytokine entry at regions of the brain (e.g. circumventricular organs) where the barrier is weak or absent to trigger the production of second messengers (e.g. prostaglandins) to neural targets (e.g. hypothalamic regions) or to induce local cytokine production (Saper \& Breder, 1994). As an alternative to the blood-borne route, several authors have pursued the possibility of direct neural signalling via the vagus nerve (Dantzer et al. 1996; Maier \& Watkins, 1999). Whatever the route used to signal the brain, most authors agree that local production of cytokines by glial cells plays an important role in the induction of sickness behaviour. This offers the best explanation of the welldocumented increase in brain levels of cytokines after peripheral administration, as well as accounting for the presence of cytokine-producing cells (glial cells) and specific binding sites throughout the brain (Vollmer-Conna et al. 1998; Maier \& Watkins, 1999). The exact mechanism responsible to translate the immune signal into a neural signal is still unclear, but appears to involve alterations in a variety of neuropeptide (e.g. corticotropin-releasing hormone $(\mathrm{CRH})$, substance $\mathrm{P}$, opioids) and neurotransmitter systems (nonadrenaline, serotonin, gamma-aminobutyric acid (GABA)) (Rothwell \& Hopkins, 1995).

\section{HUMAN STUDIES OF ACUTE SICKNESS BEHAVIOUR}

To date, very few studies have systematically examined the potential relationships between proinflammatory cytokines and mental and behavioural symptoms in sick humans. Although animal studies have contributed much to our understanding of acute sickness behaviour, they are poorly suited to examine complex behaviours and/or more subtle changes in brain function (e.g. cognitive deficits, mood alterations) that are nonetheless an integral part of sickness behaviour. Smith and colleagues $(1987,1988)$ studied cognitive performance in healthy volunteers with experimentally induced common cold (rhinovirus) or influenza, or an infusion of interferon- $\alpha$. Both influenza infections and interferon- $\alpha$ administration produced impairments in stimulus detection tasks, whereas common colds were associated with impaired hand-eye coordination. Interestingly, these cognitive performance deficits were also demonstrated in subjects with subclinical infections. Interpretation of these findings is somewhat limited by the artificial context in which they were obtained, and the use of a cytokine not generally considered one of the prototypic inflammatory cytokines. They do suggest, however, that even minor infections with agents that do not directly infect the brain, may be associated with significant impairment in cognitive performance. Moreover, such impairment appears to be related to the action of cytokines.

Significant neurocognitive deficits have also been demonstrated in a clinical sample of patients with acute infections (both Epstein-Barr virus (EBV), and influenza-like infections; Vollmer-Conna et al. 1997). In comparison to well-matched healthy controls, these patients reported a significant increase in negative affect and fatigue, and showed deficits in selective attention, response speed, pursuit tracking, and performance accuracy. A similar study recently demonstrated that patients with influenza-like illnesses were impaired on aspects of everyday memory (Capuron et al. 1999). These findings are suggestive only, as immunological correlates were not assessed and not all infections were serologically documented. What is clearly needed is the unambiguous identification of cognitive deficits associated with specific acute infections. In addition, the relationship between such deficits and the action of cytokines must be examined.

\section{CLINICAL AND PRACTICAL IMPLICATIONS OF THE BEHAVIOURAL AND MENTAL EFFECTS OF CYTOKINES}

An accumulation of evidence suggests that behavioural/mental changes typically associated with acute infections (i.e. acute sickness behaviours) are immunologically mediated and form part of the host defence. The efficacy of these behavioural disease-fighting strategies is demonstrated by the fact 
that both animals and man have survived infections and injury through evolutionary history. A better understanding of acute sickness behaviour and its underlying mechanism(s) will provide new insights into how sickness and recovery processes are organized in the brain. This may lead to the development of management practices that complement the innate disease-coping strategies, and may be particularly beneficial when dealing with viruses or drug resistant bacteria (Hart, 1988).

Several reports suggest that acute infective illness is associated with significant cognitive impairment (Smith et al. 1987; Volmer-Conna et al. 1997; Capuron et al. 1999). Yet, many patients continue with their usual daily routine throughout an illness such as influenza, glandular fever or $\mathrm{Q}$ fever. There is some indication from an ongoing large, prospective study of infective cohorts (Bennett et al. 1998) that accidents in the workplace are more prevalent in the context of acute Q fever (Lloyd, A. unpublished data). Elucidation of the full extent of behavioural and mental changes during acute infections may thus have important implications for road and work place safety.

\section{POTENTIALLY ABNORMAL MANIFESTATIONS OF SICKNESS BEHAVIOUR}

Sickness behaviour, similar to the fright-flight response, is viewed as a highly organized strategy critical to survival. However, in the same way as pathological fear and anxiety are debilitating, excessive sickness behaviour can be detrimental. Over the past decade there has been much speculation on a possible role for cytokines in the pathogenesis of neuropsychiatric syndromes, notably post-infective fatigue syndromes (e.g. Chao et al. 1991; Hickie \& Lloyd, 1995; VollmerConna et al. 1998;) and, more recently, major depression (e.g. Maes et al. 1995a; Maes 1999; Charlton, 2000).

Clinical and scientific interest in exploring the possibility of an immunological basis for neuropsychiatric disorders was initially fuelled by the striking similarity between acute sickness behaviours and key symptoms reported in depression and fatigue syndromes (i.e. loss of appetite, malaise, psychomotor slowing, altered sleep, fatigue, anhedonia, depressed affect and cognitive impairment). In addition, the discovery that psychosocial stressors can activate the inflammatory response system has lent more credence to a proposed role for pro-inflammatory cytokines in the pathogenesis of stress-related disorders such as depression (Maes, 1999).

Research examining immunological correlates of depression has produced evidence consistent with the notion of immune activation, including elevated leukocyte counts and activation markers, increased production of pro-inflammatory cytokines and acute phase proteins. Moreover, tricyclic antidepressants and serotonin reuptake inhibitors appear to suppress these inflammatory responses (Maes et al. 1995a, for review). On the other hand, there is substantial evidence documenting immunosuppression and increased susceptibility to disease in patients with major depression (Herbert \& Cohen, 1993). It has been suggested that the simultaneous signs of immune activation and suppression in depression may be reconciled by reference to the action of multiple feedback systems generated to contain the immune response or T cell exhaustion (Maes et al. 1995b).

The emergence of immunological hypotheses for neuropsychiatric disorders, such as depression, challenges traditional pathophysiological views, and provides a fresh perspective on symptomatology (e.g. depression as a variant of sickness behaviour). However, the data to date are not sufficient to determine whether altered levels of pro-inflammatory cytokines play an aetiological role, are the consequence of depression, or merely reflect an epiphenomenon. Interpretation of the available evidence is limited by a variety of methodological problems including the predominant reliance on cross-sectional, correlational designs, one-off sampling and the inevitable heterogeneity of subject samples. An additional complication inherent in this type of research lies in the attempt to establish associations between centrally-mediated symptoms and measurements obtained from peripheral blood samples. Although such endeavours are justified, in principle, by the bi-directional nature of the connection between the brain and the immune system, it is unlikely that clear and definitive answers about the role of specific circulating cytokines in the production of specific psychopathological symptoms can be established in this way.

Understanding potentially abnormal variants of a natural phenomenon clearly requires an in- 
depth knowledge of the phenomenon in question. Therefore, a systematic study of normal sickness behaviour in humans (examining the full spectrum, development and immunological mediators of symptoms during acute infections) is needed to construct a sound knowledge-base enabling identification and evaluation of inappropriate or excessive manifestations of this phenomenon. Moreover, such knowledge is essential in light of the fact that the manipulation of cytokine systems is rapidly developing into a new area of therapeutics (Dinarello, 1997), and which may have unanticipated neurobehavioural consequences.

UTÉ VOLLMER-CONNA

I wish to thank Professor Gordon Parker for invaluable advice and encouragement throughout the preparation of this paper.

\section{REFERENCES}

Ader, R., Felten, D. L. \& Cohen, N. (1991). Psychoneuroimmunology, 2nd edn. Academic Press: San Diego, CA.

Banks, W. A., Oritz, L., Plotkin, S. R. \& Kastin, A. J. (1991) Human interleukin (IL) 1 alpha, murine IL-1 alpha, and murine IL-1 beta are transported from blood to brain in the mouse by a shared saturable mechanism. Journal of Pharmacology and Experimental Therapeutics 259, 988-996.

Beck, G., Cooper, E. L., Hobicht, G. S. \& Marchalonis, J. J. (1994). Primordial immunity: foundations for the vertebrate immune system. Annals of the New York Academy of Sciences 712, 206-212.

Bennett, B. K., Hickie, I. B., Vollmer-Conna, U. S., Quigley, B., Brennan, C. M., Wakefield, D., Douglas, M. P., Hansen, G. R., Tahmindjis, A. J. \& Lloyd, A. R. (1998). The relationship between fatigue, psychological and immunological variables in acute infectious illness. Australian and New Zealand Journal of Psychiatry 32, 180-186.

Bluthe, R. M., Michaud, B., Poli, V., Bernay, F., Parnet, P. \& Dantzer, R. (1998). Interleukin-6 is active only in presence of other proinflammatory cytokines to induce sickness behaviour. Neuroimmunomodulation $\mathbf{5}, 7$.

Capuron, L., Lamarque, D., Dantzer, R. \& Goodall, G. (1999). Attentional and mnemonic deficits associated with infectious diseases in humans. Psychological Medicine 29, 291-297.

Chao, C. C., Janoff, E. N., Hu, S., Thomas, K., Gallagher, M., Tsang, M. \& Peterson, P. K. (1991). Altered cytokine release in peripheral blood mononuclear cell cultures from patients with chronic fatigue syndrome. Cytokine 3, 292-298.

Charlton, B. G. (2000). The malaise theory of depression: major depressive disorder is sickness behaviour and antidepressants are analgesics. Medical Hypotheses 54, 126-130.

Clatworthy, A. L. (1996). A simple system approach to neuralimmune communication. Comparative Biochemistry and Physiology 115A, $1-10$.

Dantzer, R., Bluthe, R.-M., Aubert, A., Goodall, G., Bret-Dibat, JL., Kent, S., Goujon, E., Laye, S., Parnet, P. \& Kelley, K. W. (1996). Cytokine actions on behavior. In Cytokines in the Nervous System (ed. N. J. Rothwell), pp. 117-144. R. G. Landes Co.: New York.

Dinarello, C. A. (1997). Proinflammatory and anti-inflammatory cytokines as mediators in the pathogenesis of septic shock. Chest 112, 312S-329S.

Dinarello, C. A., Renfer, L. \& Wolff, S. M. (1977). Human leukocytic pyrogen: purification and development of a radioummunoassay. Proceedings of the National Academy of Science USA 74, 4624.

Dunn, A. J. (1995). Interactions between the nervous system and the immune system: implications for psychopharmacology. In Psychopharmacology: The fourth Generation of Progress (ed. F. E. Bloom and D. J. Kupfer), pp. 719-733. Raven Press: New York.

Hart, B. L. (1988). Biological basis of the behavior of sick animals. Neuroscience and Biobehavioral Reviews 12, 123-137.

Herbert, I. \& Cohen, S. (1993). Depression and immunity: a metaanalytic review. Psychological Bulletin 113, 472-486.
Hickie, I. \& Lloyd, A. (1995). Are cytokines associated with neuropsychiatric syndromes in humans? International Journal of Immunopharmacology 17, 677-683.

Kent, S., Bluthe, R. M. \& Kelley, K. W. (1992). Sickness behaviour as a new target for drug development. Trends in Pharmacological Sciences 13, 24-28.

Kluger, M. J. (1979). Fever: Its Biology, Evolution and Function. Princeton University Press: Princeton, NJ.

Kusnekov, A. W. \& Rabin, B. S. (1994). Stressor-induced alterations of immune function: mechanisms and issues. International Archives of Allergy and Immunology 105, 107-121.

Lloyd, A., Hickie, I., Hickie, C., Dwyer, J. \& Wakefield, D. (1992). Cell-mediated immunity in patients with chronic fatigue syndrome, healthy control subjects and patients with major depression. Clinical and Experimental Immunology 87, 76-79.

Maes, M. (1999). Psychological stress, cytokines and the inflammatory response system. Current Opinion in Psychiatry 12, 695-700.

Maes, M., Smith, R. \& Scharpe, S. (1995a). The monocyte-T lymphocyte hypothesis of major depression. Psychoneuroendocrinology 20, 111-116.

Maes, M., Meltzer, H. Y., Bosmans, E., Bergmans, R., Vandoolaeghe, E., Ranjan, R. \& Desynder, R. (1995 b). Increased plasma concentrations of interleukin-6, soluble interleukin-6, soluble interleukin-2 and transferrin receptor in major depression. Journal of Affective Disorders 34, 301-309.

Maier, S. F. \& Watkins, L. R. (1995). Intracerebroventricular intereukin-1 receptor antagonist blocks the enhancement of fear conditioning and interference with escape learning produced by inescabable shock. Brain Research 695, 279-286.

Maier, S. F. \& Watkins, L. R. (1998). Cytokines for psychologists: implications of bi-directional immune-to-brain communication for understanding behavior, mood, and cognition. Psychological Review 105, 83-107.

Maier, S. F. \& Watkins, L. R. (1999). Bidirectional communication between the brain and the immune system: implications for behaviour. Animal Behaviour 57, 741-751.

Maier, S. F., Watkins, L. R. \& Fleshner, M. (1994). Psychoneuroimmunology: the interface between behaviour, brain, and immunity. American Psychologist 49, 1004-1017.

Mastorakos, G., Chrousos, G. P. \& Weber, J. S. (1993). Recombinant interleukin-6 activates the hypothalamic-pituitary-adrenal axis in humans. Journal of Clinical Endocrinology and Metabolism 77, 1690-1694.

Papanicolaou, D. A. (1998). The pathophysiologic roles of interleukin-6 in human disease. Annals of Internal Medicine 128, $127-137$.

Renault, N. J. \& Hoofnagel, J. H. (1989). Side effects of alpha interferon. Seminars in Liver Disease 9, 273-277.

Rothwell, N. J. \& Hopkins, S. J. (1995). Cytokines and the nervous system: actions and mechanisms of action. Trends in Neuroscience 18, 130-136.

Rozlog, L. A., Kiecolt-Glaser, J. K., Marucha, P. T., Sheridan, J. F. \& Glaser, R. (1999). Stress and immunity: implications for viral disease and would healing. Journal of Periodontology 70, 786-792. 
Saper, C. B. \& Breder, C. D. (1994). The neurologic basis of fever. New England Journal of Medicine 330, 1880-1886.

Shintani, F., Nakaki, T., Kanba, S., Sato, K., Yagi, G., Shiozawa, M., Aiso, S., Kato, R. \& Asai, M. (1995). Involvement of interleukin-1 in immobilization stress-induced increase in plasma adrenocorticotropic hormone and in the release of hypothalamic monoamines in the rat. Journal of Neuroscience 15, 1961-1970.

Smith, A. P., Tyrrell, D. A. J., Al-Nakib, W., Coyle, K. B., Donovan, C. B., Higgins, P. G. \& Willman, J.S. (1987). Effects of experimentally induced respiratory virus infections and illness on psychomotor performance. Neuropsychology 18, 144-148.

Smith, A., Tyrrell, D., Coyle, K. \& Higgins, P. (1988). Effects of interferon alpha on performance in man: a preliminary report. Psychopharmacology 96, 414-416.
Takaki, A., Huang, Q. H., Somogyvari-Vigh, A. \& Arimura, A. (1994). Immobilization stress may increase plasma IL-6 activity via central and peripheral catecholamines. Neuroimmunomodulation $\mathbf{1}$, 335-342.

Tringali, G., Dello Russo, C., Preziosi, P. \& Navarra, P. (2000). Interleukin-1 in the central nervous system: from physiology to pathology. Thérapie 55, 171-175.

Vollmer-Conna, U., Wakefield, D., Lloyd, A., Hickie, I., Lemon, J., Bird, K. D. \& Westbrook, R. F. (1997). Cognitive deficits in patients suffering from chronic fatigue syndrome, acute infective illness or depression. British Journal of Psychiatry 171, 377-381. Vollmer-Conna, U., Lloyd, A., Hickie, I. \& Wakefield, D. (1998) Chronic fatigue syndrome: an immunological perspective Australian and New Zealand Journal of Psychiatry 32, 523-527. 